\title{
EFEITOS DA ACUPUNTURA NO DESEMPENHO MOTOR DE ATLETAS
}

\author{
Heloisa Belmiro, Universidade Estadual de Londrina - UEL, Londrina, Paraná - \\ Brasil \\ Daniel Vicentini de Oliveira, Centro Universitário de Maringá - CESUMAR, Maringá, \\ Paraná-Brasil \\ Carmen Maria Camilotti, Universidade Estadual de Londrina - UEL, Londrina, \\ Paraná-Brasil
}

\section{RESUMO}

A acupuntura é um recurso terapêutico do sistema médico conhecido no mundo ocidental como Medicina Tradicional Chinesa (MTC) Ela visa à terapia e à cura das doenças através da aplicação de agulhas e moxas, além de outras técnicas. Atualmente, tem sido utilizada para o tratamento de dores crônicas e agudas de diversas origens, para controle da dor durante alguns procedimentos cirúrgicos, prevenção e tratamento de doenças, dores musculoesqueléticas, promover a normalização funcional do organismo e tem sido utilizada por atletas, profissionais e amadores para modular o bem-estar físico, para o alcance de um nível mais alto de performance competitiva e para um melhor treinamento. Apesar de ser uma área ainda pouco explorada e estudada, os resultados dos presentes estudos, já justificam o uso da acupuntura como parte integrante de uma rotina atlética de condicionamento físico, e um acupunturista desportivo já deveria estar presente nas equipes desportivas de alto rendimento, a fim de melhorar a performance final dos atletas.

Palavras-Chave: Desempenho atlético; Esporte; Terapia por acupuntura.

\section{EFFECTS OF ACUPUNCTURE IN MOTOR PERFORMANCE OF ATHLETES}

\begin{abstract}
Acupuncture is a therapeutic medical system in the Western world known as Traditional Chinese Medicine (TCM) aims It aims to therapy and healing of diseases through the application of needles and moxas, and other techniques. Currently, it has been used to treat chronic and acute pain of various origins, to control pain during some surgical procedures, prevention and treatment of diseases., Musculoskeletal pain, promote functional normalization of the body and has been used by athletes, professional and amateurs to modulate the physical well-being, to reach a higher level of competitive performance and better training. Despite being an area little explored and studied, the results of these studies have justified the use of acupuncture as part of a routine athletic Conexões: revista da Faculdade de Educação Física da UNICAMP, Campinas, v. 11, n. 3, p. 176-191, jul./set. 2013. ISSN: 1983-9030


fitness, and sports an acupuncturist should already be present in high performance sports teams, the order to enhance the end performance of athletes.

Key-Words: Athletic performance; Sport; Acupuncture therapy.

\section{EFECTOS DE LA ACUPUNTURA EN ATLETAS DE RENDIMIENTO DEL MOTOR}

\section{RESUMEN}

La acupuntura es un sistema médico terapéutico conocido en Occidente como la Medicina Tradicional China (MTC) tiene como objetivo Apunta a la terapia y curación de las enfermedades mediante la aplicación de agujas y moxas, y otras técnicas. En la actualidad, se ha utilizado para el tratamiento del dolor agudo y crónico de diversos orígenes, para controlar el dolor durante algunos procedimientos quirúrgicos, la prevención y tratamiento de enfermedades., Dolor musculoesquelético, promover la normalización funcional del organismo y se ha utilizado por atletas, profesionales y amateurs para modular el bienestar físico, para alcanzar un nivel más alto de desempeño competitivo y el mejor entrenamiento. A pesar de ser un área poco explorada y estudiada, los resultados de estos estudios, ya que justifica el uso de la acupuntura como parte de una rutina de fitness atlético, deportivo y un acupunturista ya debería estar presente en los equipos deportivos de alto rendimiento, el mejorar el rendimiento final de los atletas.

Palabras-Clave: Rendimiento atlético; Deporte; Terapia de acupuntura 


\section{INTRODUÇÃO}

Nos dias atuais, em função do ritmo de vida corrido das grandes metrópoles e das transformações que vêm ocorrendo em nossa sociedade, é cada vez mais comum nos depararmos com os termos terapias corporais alternativas, terapias holísticas ou mesmo práticas corporais. Tais práticas se configuram como opções diferentes daquilo que é comum, tradicional, principalmente em termos de tratamentos de saúde, alimentação, ginásticas, vestimentas, ou seja, um estilo de vida alternativo ao convencional. ${ }^{1-2}$

A acupuntura é uma das dessas modalidades alternativas de tratamento do arsenal terapêutico tradicional chinês. Atualmente está sendo usada em mais de 140 países como uma terapia alternativa com poucos efeitos colaterais, de simples manipulação e baixo custo. Ela está ao alcance dos profissionais de saúde de formação variada, de acordo com sua área de atuação profissional. ${ }^{3-5}$

Essa técnica é recomendada pela Organização Mundial da Saúde (OMS) para o tratamento de 41 doenças, como por exemplo, asma e dores em geral. Ademais, tem sido utilizada para o tratamento e preparo de atletas.

O presente estudo teve como objetivo compilar, organizar e discutir sobre pesquisas científicas a respeito de tratamentos por acupuntura realizados em atletas.

Trata-se de uma pesquisa bibliográfica, em que o levantamento dos dados foi realizado através de artigos científicos selecionados nas bases de dados Scielo, Lilacs e MEDLINE, através das seguintes palavras chave: acupuntura, acupuntura no esporte e acupuntura em atletas, tendo como critérios de inclusão artigos completos, publicados em revistas indexadas, independente do ano e do delineamento de estudo, se pesquisa de revisão ou de intervenção, nas línguas portuguesa e inglesa, além de livros da biblioteca da Universidade Estadual de Londrina (UEL), Paraná, no período de Janeiro a Outubro de 2011.

Conexões: revista da Faculdade de Educação Física da UNICAMP, Campinas, v. 11, n. 3, p. 176-191, jul./set. 2013. ISSN: 1983-9030 


\section{REVISÃO BIBLIOGRAFICA}

\section{ACUPUNTURA}

A acupuntura é um recurso terapêutico do sistema médico conhecido no mundo ocidental como Medicina Tradicional Chinesa (MTC). Ela visa à terapia e à cura das doenças através da aplicação de agulhas e moxas, além de outras técnicas. ${ }^{6}$

Uma outra definição seria que a acupuntura se assemelha-se a penetração da pele com agulhas de aço inoxidável que estimulam o tecido manual, térmica e eletricamente. A palavra é derivada dos radicais latinos acus e pungere, que significam agulha e puncionar, respectivamente. ${ }^{7}$

Segundo o conceito de racionalidades médicas, temos um sistema médico quando este apresenta características próprias e originais quanto as seguintes dimensões fundamentais: morfologia, dinâmica vital, diagnose, doutrina e terapêutica. ${ }^{8}$

A MTC atende a todos estes quesitos, apresentando, por exemplo: na morfologia, o sistema de canais e colaterais (jingluo); na dinâmica vital, os sistemas internos (zangfu); na diagnose, a Pulsologia Chinesa; na doutrina, os Oito Princípios (ba gang) e os Cinco Elementos (wu xing), e na terapêutica, a acupuntura e a moxabustão (zhenjiu), dentre outros recursos. ${ }^{9}$

Segundo a tradição chinesa os pontos de acupuntura estão localizados ao longo de canais específicos cuja puntura nesses locais facilita e restaura o fluxo de energia do corpo. Em outras palavras, ela controla o fluxo de energia através dos canais que estabelecem o equilíbrio do corpo (Ying e Yang), em que a doença é um reflexo da perda de harmonia. ${ }^{10-12}$ Assim, essa técnica tem sido usada para o tratamento de desordens clínicas na China por mais de cinco mil anos. ${ }^{12}$

Atualmente, tem sido utilizada para o tratamento de dores crônicas e agudas de diversas origens e, para controle da dor durante alguns procedimentos cirúrgicos, além de seu uso para a prevenção e tratamento de doenças. É indicada para dores 
musculoesqueléticas como para promover a normalização funcional do organismo. ${ }^{10,13}$

A acupuntura, além de ser aplicada na prevenção do equilíbrio energético do organismo humano, dos animais e das plantas e na terapêutica de doenças, é também utilizada no Extremo Oriente, desde tempos imemoriais, para "tonificar" ou "sedar" os músculos dos esportistas trabalhadores. ${ }^{14}$

Os trabalhos sobre o tratamento da acupuntura no esporte ainda são escassos. No entanto a utilização da acupuntura tem se mostrado benéfica para um número significativo de pessoas. Embora ainda não tenha sido explicada a relação entre os meridianos e as vias nervosas anatômicas. ${ }^{15-16}$

Existem diferentes variações de técnica da acupuntura, as quais podemos citar a eletroacupuntura (envia uma corrente elétrica através das agulhas para estimular os pontos de pressão durante a sessão); a sonoacupuntura (aplica ondas sonoras aos pontos de acupuntura. As vibrações estimulam pontos de pressão de maneira mais sutil do que as agulhas; a sonoacupuntura costuma ser combinada com a acupuntura); a acupressão (segue os mesmos princípios que a acupuntura, mas utiliza pressão em vez de agulhas. O terapeuta utiliza seus dedos para pressionar os pontos de pressão dos pacientes, e mantém essa pressão por alguns segundos). ${ }^{17}$

Existe também as técnicas de moxibustão, que utiliza o calor para estimular os pontos da acupuntura. $\mathrm{O}$ calor é gerado pela queima de uma erva chamada moxa, que vem da artemísia. Há dois tipos de moxibustão, a direta e indireta. Na moxibustão direta, um pedaço da erva, do tamanho de um grão de arroz, é colocado diretamente sobre a pele e queimado sobre um ponto da acupuntura. Como isso pode ser doloroso e deixar cicatrizes, vários praticantes preferem a moxibustão indireta, na qual o pedaço de moxa é embalado em um papel, aceso e mantido próximo à pele. Algumas vezes, a moxa é usada para embalar agulhas de acupuntura e acesa para adicionar estímulos extras ao tratamento de acupuntura. ${ }^{17}$

Conexões: revista da Faculdade de Educação Física da UNICAMP, Campinas, v. 11, n. 3, p. 176-191, jul./set. 2013. ISSN: 1983-9030 


\section{ACUPUNTURA NO ESPORTE}

Há poucos estudos experimentais sobre a acupuntura como recurso para a melhora da performance física e seus achados são contraditórios. Pouca atenção científica tem sido voltada para o estudo dos efeitos da acupuntura nas respostas fisiológicas ao exercício e, nesse sentido, também poucas pesquisas publicadas têm estudado, especificamente, atletas. $^{10}$

A acupuntura tem sido utilizada por atletas, profissionais e amadores para modular o bem-estar físico, para o alcance de um nível mais alto de performance competitiva e para um melhor treinamento. ${ }^{10}$

Em contrapartida, a estimulação de pontos de acupuntura específicos ainda tem sido sugerido para melhorar a performance física e esportiva, as qualidades físicas básicas e a plasticidade muscular. ${ }^{10,17}$

Como os atletas praticamente já utilizaram diversas maneiras para a melhora de sua performance, a acupuntura é uma forma de complementar e auxiliar na melhora do desempenho e bem estar físico, que, consequentemente, ajuda na melhoria de treinamentos e jogos. ${ }^{9}$

O bom desempenho de um atleta requer higidez funcional dos seus vários sistemas orgânicos. A sobrecarga do sistema músculo esquelético em resposta à intensa atividade física durante o treinamento, às competições ou apresentações, exige do corpo níveis de força muscular, de amplitude articular e transferência de peso muito acima dos fisiológicos. ${ }^{18}$

Os movimentos corporais realizados no esporte sofrem mudanças inesperadas e, quando são associados a interrupções rápidas, bruscas e de grande impacto, podem levar a perda de acomodação das estruturas osteoarticulares e miotendiosas. ${ }^{18}$

Em um estudo ${ }^{19}$ realizado com ratos, a relação da acupuntura com o exercício, 
revelando que os efeitos supressivos da acupuntura sobre a 5 HT (5 hidroxitriptamina) e a expressão TPH (triptoflano hidroxilase) na rafe dorsal das cobaias seria um dos mecanismos ergogênicos da acupuntura, pois comprovou-se que a concentração aumentada de 5 HT impede a performance do exercícios em seres humanos e ratos.

Concluiu-se em estudo ${ }^{20}$ que, nos países asiáticos um tratamento de acupuntura traz aos atletas um estado de bem estar físico e com isso melhora de sua performance nas respectivas modalidades.

Estudos mostraram que o tratamento com acupuntura produziu vários efeitos como: alívio da tensão muscular, aumento do fluxo sanguíneo local, aumento no limiar de dor e melhor modulação do sistema nervoso autônomo. ${ }^{13}$

Os artigos pesquisados encontram-se sistematizados no Quadro 1 e 2 e são discutidos com mais detalhes, a seguir. 
Quadro 1 - Revisão bibliográfica sobre acupuntura em atletas de 1992 a 2005

\begin{tabular}{|c|c|c|c|c|c|}
\hline REFERÊENCIA & AMOSTRA & TÉCNICA & ACUPONTOS & AVALIAÇÃO & RESULTADOS \\
\hline $\begin{array}{l}\text { HABER, D. E. et al. } \\
\text { Influence of acupuncture } \\
\text { on physical performance } \\
\text { capacity } \\
\text { haemodyamic parameters. } \\
\text { Int. J. Sports Med., v. 13, } \\
\text { 1992. }\end{array}$ & $\begin{array}{l}36 \text { homens } \\
\text { jovens } \\
\text { saudáveis }\end{array}$ & $\begin{array}{l}\text { Acupuntura } \\
\text { Sistêmica }\end{array}$ & $\begin{array}{lr}\text { VG20, } & \text { KG15, } \\
\text { B38, } & \text { Le13, } \\
\text { KS6, } & \text { M36, } \\
\text { MP6. } & \end{array}$ & $\begin{array}{lr}\text { Capacidade } & \text { de } \\
\text { desempenho } & \text { físico; } \\
\text { regulação } & \text { da } \\
\text { frequência cardíaca } & \text { e } \\
\text { pressão arterial. } & \end{array}$ & $\begin{array}{l}\text { Aumento significante da } \\
\text { capacidade máxima de } \\
\text { performance e também o } \\
\text { desempenho físico no } \\
\text { limiar anaeróbio }\end{array}$ \\
\hline $\begin{array}{l}\text { GENG, L. J. et al. } \\
\text { Investigation on the } \\
\text { Effects of Ear Acupressure } \\
\text { on Exercise-Induced } \\
\text { Lactic Acid Levels and the } \\
\text { Implications for Athletic } \\
\text { Training. American } \\
\text { Journal Of Acupuncture, v. } \\
\text { 23, n. 4, 1995. }\end{array}$ & $\begin{array}{l}12 \text { homens } \\
\text { saudáveis } \\
\text { estudantes de } \\
\text { educação } \\
\text { física, com } \\
\text { idade entre } 19 \\
\text { e } 25 \text { anos. }\end{array}$ & $\begin{array}{l}\text { Acupuntura } \\
\text { Auricular }\end{array}$ & $\begin{array}{l}\text { Fígado Pulmão, } \\
\text { Subcórtex, } \\
\text { Glândulas } \\
\text { endócrinas, } \\
\text { Triplo } \\
\text { Aquecedor. }\end{array}$ & $\begin{array}{l}\text { Efeitos da acupuntura } \\
\text { auricular nos níveis de } \\
\text { ácido lático sanguíneo, } \\
\text { induzidor pelo } \\
\text { exercício }\left(\mathrm{VO}^{2} \max \right)- \\
\text { esteira ergométrica. }\end{array}$ & $\begin{array}{l}\text { Aumento da captação de } \\
\text { oxigênio e diminuição dos } \\
\text { níveis de ácido láctico } \\
\text { sanguíneo, pós exercício. }\end{array}$ \\
\hline $\begin{array}{l}\text { BOOP-LIMOGE, C. L' } \\
\text { acupunture permet-elle d' } \\
\text { ameliorer les } \\
\text { performances sportives } \\
\text { etude personnelle a propos } \\
\text { de } 35 \text { sportifs de hault } \\
\text { niveau. These Medicine, } \\
1998 .\end{array}$ & $\begin{array}{l}\text { Cinco } \\
\text { homens e } \\
\text { cinco } \\
\text { mulheres } \\
\text { atletas de } \\
\text { diferentes } \\
\text { modalidades }\end{array}$ & $\begin{array}{l}\text { Acupuntura } \\
\text { Sistêmica }\end{array}$ & F5, TA15, B58 & Performance Física & $\begin{array}{l}\text { Eficaz na performance } \\
\text { física. }\end{array}$ \\
\hline $\begin{array}{l}\text { AKIMOTO, T. et al. } \\
\text { Acupuncture } \\
\text { responses of immunologic } \\
\text { and endocrine markers } \\
\text { during competion. } \\
\text { Medicie \& Science In } \\
\text { Sports \& Exercise, } 2003\end{array}$ & $\begin{array}{l}21 \text { mulheres } \\
\text { atletas de elite } \\
\text { de futebol } \\
\text { feminino }\end{array}$ & $\begin{array}{l}\text { Acupuntura } \\
\text { Sistêmica }\end{array}$ & P4, E36, E6, F6 & $\begin{array}{l}\text { Bem estar físico } \\
\text { durante período de } \\
\text { competição, níveis da } \\
\text { imunoglobulina } \\
\text { (SigA) e níveis de } \\
\text { cortisol, ambos via } \\
\text { salivar }\end{array}$ & $\begin{array}{l}\text { A diminuição de dos } \\
\text { níveis de SigA salivar, } \\
\text { induzida pelo exercício, e } \\
\text { o aumento dos níveis de } \\
\text { cortisol salivares, foram } \\
\text { inibidos pela acupuntura. } \\
\text { Melhorou a avaliação } \\
\text { subjetiva de tensão } \\
\text { muscular e fadiga; A } \\
\text { pontuação POMS foi } \\
\text { modulada } \\
\text { acupuntura. }\end{array}$ \\
\hline $\begin{array}{l}\text { LUNA, M. P. et al. Efeitos } \\
\text { da acupuntura } \\
\text { performance de atletas } \\
\text { velocistas de } \\
\text { rendimento do Rio de } \\
\text { Janeiro. Fitness \& } \\
\text { Performance, v. 4, n. 4, } \\
2005 \text {. }\end{array}$ & $\begin{array}{l}16 \text { atletas de } \\
\text { atletismo } \\
\text { (velocistas) } \\
\text { com idade } \\
\text { entre } 16 \text { e } 27 \\
\text { anos }\end{array}$ & $\begin{array}{l}\text { Acupuntura } \\
\text { Sistêmica }\end{array}$ & $\begin{array}{l}\text { B62, } \text { BP3, } \\
\text { VB34, R3, F8, } \\
\text { F3, F1, E36, } \\
\text { ID3 }\end{array}$ & $\begin{array}{lr}\text { Variáveis de força } \\
\text { máxima dinâica, força } \\
\text { explosiva, resistência } \\
\text { anaeróbica } \\
\text { velocidade. } \\
\text { Desempenho } \\
\text { corredores velocistas } \\
\text { de alto rendimento, } \\
\text { durante o "período } \\
\text { transição" }\end{array}$ & $\begin{array}{l}\text { Melhora das marcas em } \\
\text { competições. }\end{array}$ \\
\hline
\end{tabular}

Conexões: revista da Faculdade de Educação Física da UNICAMP, Campinas, v. 11, n. 3, p. 176-191, jul./set. 2013. 
Quadro 2 - Revisão bibliográfica sobre acupuntura em atletas de 2008 a 2010

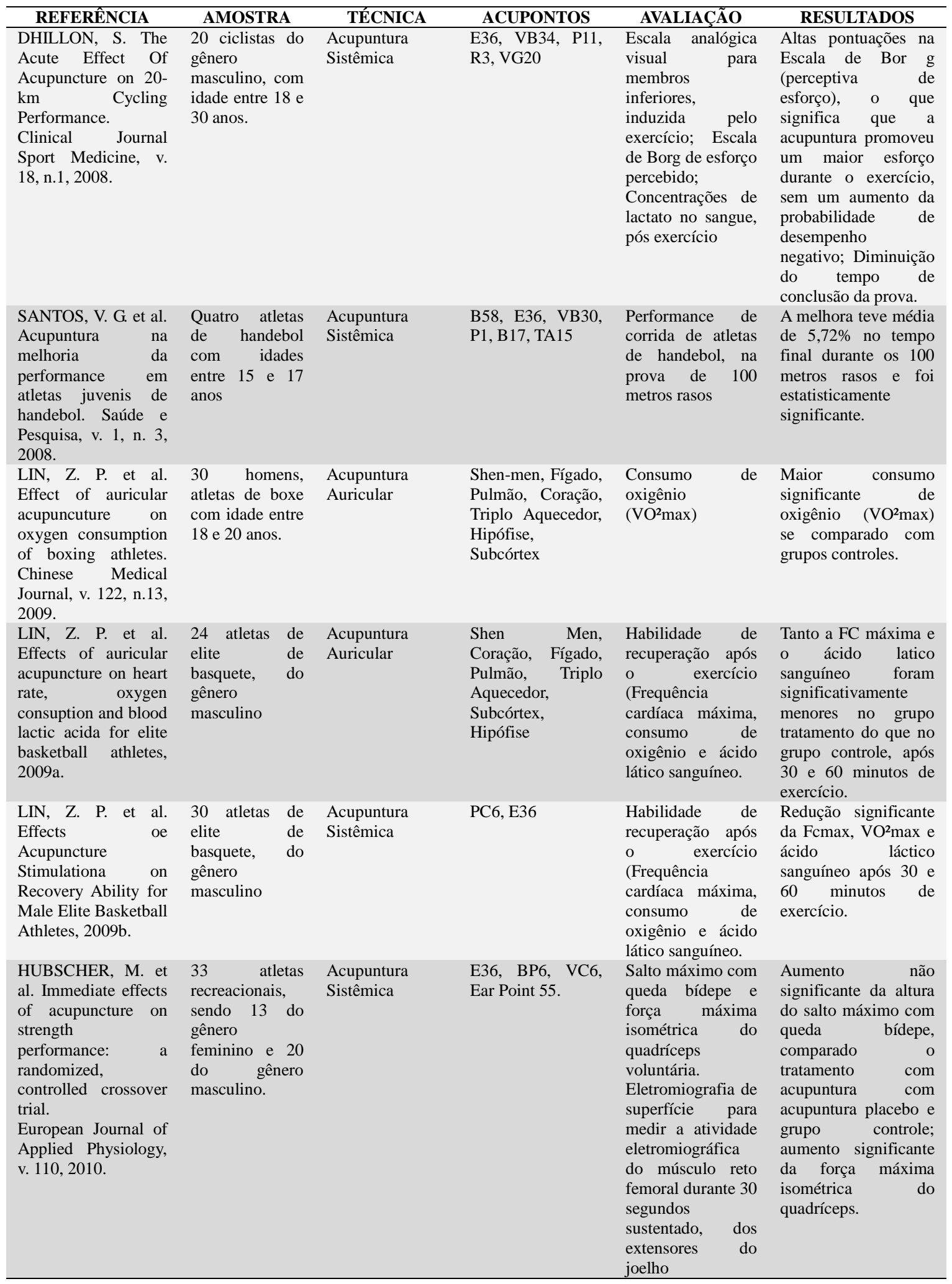

Conexões: revista da Faculdade de Educação Física da UNICAMP, Campinas, v. 11, n. 3, p. 176-191, jul./set. 2013. 


\section{ESTUDOS EXPERIMENTAIS}

Em um estudo ${ }^{9}$ constatou-se a eficácia da acupuntura na melhoria da performance de corrida em atletas de handebol, utilizando os pontos B58, E36, VB30, P1, B17, TA15. A melhora teve média de $5,72 \%$ no tempo final durante os 100 metros rasos e foi estatisticamente significante. Essa porcentagem na prática indica um ganho representativo no desempenho e performance dos atletas de handebol.

Outro estudo, ${ }^{10}$ procurou responder quais os efeitos da acupuntura sobre as variáveis de força máxima dinâmica, força explosiva, resistência anaeróbica e velocidade. Além disso, também procurou testar a hipótese de que a acupuntura melhoraria essas variáveis e, consequentemente, o desempenho da população estudada, composta de corredores velocistas de alto rendimento do Rio de Janeiro, durante o período chamado de "período de transição", quando só se espera que um atleta mantenha os ganhos conquistados ao longo do ciclo de condicionamento e na temporada de competições.

O estudo acima apresentou um maior enfoque para o meridiano do fígado, com os pontos F8, F3, F1. As funções energéticas do fígado são armazenar o sangue, controlar a dispersão, a drenagem e determinar as condições dos tendões e dos ligamentos, e também tem seu papel na parte emocional, se essa função estiver normal, o atleta será alegre, mas se estiver anormal, ele poderá sofrer com depressão, irritabilidade, insônia, sonhos perturbados, entre outras muitas funções desse órgão importante dentro da MTC, o fígado pode ser visto como "o general das forças armadas".

Esse estudo mostrou preliminarmente que a acupuntura impactou positivamente a população estudada, pois os resultados sugerem que foi revertida em curto prazo, em dois meses, uma tendência histórica de piora das marcas dessa população em competições.

Em outra pesquisa, ${ }^{21} 36$ homens saudáveis foram divididos em três grupos (acupuntura sistêmica, grupo controle e grupo acupuntura placebo). Os resultados foram positivos para a performance física e para os parâmetros hemodinâmicos. Os autores realizaram 
durante cinco semanas, uma sessão de acupuntura a cada semana, nos pontos VG20, VC15, B43, F13, CS6, E36, BP6.

Perceberam que o limiar anaeróbico melhorou em média 6,62\% e a performance máxima melhorou $7,15 \%$ no grupo que recebeu acupuntura sistêmica. No grupo controle e placebo não houve mudança significativa.

Em uma tese de medicina, ${ }^{22}$ realizada em Strasbourg, estudou 35 atletas de alto nível, de diferentes modalidades, aplicando acupuntura sistêmica nos pontos F5, TA15, B58, tendo como resultado uma melhora muito significante na performance da amostra estudada.

Em 2003, foi realizada uma pesquisa ${ }^{23}$ com 21 atletas de elite de futebol feminino, dividas em dois grupos. O primeiro grupo, com 9 atletas, recebeu tratamento com acupuntura sistemica e o segundo grupo, com 12 atletas, como grupo controle. No grupo que recebeu tratamento, foram utilizados os pontos LI4, E36, por 20 minutos, e o E6, LU6 por 15 minutos, quatro horas antes de cada jogo, no período noturno, durante o período da competição. Foi avaliado a secreção da imunoglobulina A na saliva (SigA salivar), nível de cortisol na saliva, avaliação subjetiva de bem-estar físico e perfil dos estados de humor (POMS). O grupo que recebeu tratamento teve como resultado a diminuição de níveis de SigA salivar, induzida pelo exercício, e o aumento de níveis de cortisol salivares. Houve melhora da avaliação subjetiva de tensão muscular e fadiga; a pontuação POMS foi modulada pela acupuntura.

Foi pesquisado também ${ }^{23}$ os efeitos da acupuntura auricular na frequência cardíaca (FCmáx), consumo de oxigênio $\left(\mathrm{VO}^{2} \mathrm{max}\right)$ e ácido lático sanguíneo em atletas de elite de basquete. Os 24 atletas foram divididos em dois grupos (Grupo 1: acupuntura auricular; Grupo 2; Grupo controle, com 12 atletas cada grupo). Cada sujeito em ambos os grupos, pedalam na bicicleta estacionária até a exaustão. A FCmáx, o $\mathrm{VO}^{2}$ máx e o ácido lático sanguíneo foram mensurados durante o período de descanso após aquecimento e no exercício, após cinco, 30 e 60 minutos. Os resultados mostraram que, 
tanto a FC e o ácido lático sanguíneo foram significantemente mais baixos do que aqueles no grupo controle, no após 30 e 60 minutos de exercício.

Os atletas conseguiram melhora na FC máxima e uma diminuição significativa no ácido lático sanguíneo. Há um consenso entre os autores para o uso do ponto E36, que foi citado na maioria dos trabalhos encontrados, sendo um ponto muito importante dentro de diferentes tratamentos com acupuntura, ele fortalece o qi e o yang, nutre o sangue, nutre o yin, tranqüiliza o shen entre outras funções, e também mostrou a sua importância no meio dos esportes.

O mesmo autor acima realizou outro estudo, ${ }^{24}$ também com atletas homens, de basquete, porém com o uso da acupuntura sistêmica, no ano de 2002. Foram selecionados 30 atletas, divididos em três grupos (acupuntura sistêmica, grupo sham, e grupo controle). No grupo de acupuntura sistêmica, foram utilizados os pontos PC6, E36. Durante a pesquisa, cada sujeito realizou ensaios separados na esteira ergométrica, por 15 minutos e foram analisados, a FCmáx, o $\mathrm{VO}^{2}$ max e o ácido lático sanguíneo. Estas variáveis foram medidas durante o período de descanso e os três pontos de recuperação de tempo (cinco, 30 e 60 minutos) após o exercício. Como resposta, obteve-se uma redução significante da $\mathrm{FCmáx} \mathrm{VO}^{2} \max$ e ácido láctico sanguíneo após 30 e 60 minutos de exercício, igual quando se aplicou a acupuntura auricular em atletas do mesmo esporte.

Foi avaliado o efeito da acupuntura na performance de ciclistas. ${ }^{25} \mathrm{~A}$ amostra constava de 20 atletas do gênero masculino, e foi aplicado nos mesmos, acupuntura sistêmica (E36, VB34, F11, R3, VG20), acupuntura sham e também nenhuma intervenção, antes de cada teste, do total de 3 ( 1 por semana), onde os atletas deveriam pedalar em uma bicicleta estacionária por $20 \mathrm{~km}$, na maior velocidade possível. Analisaram a escala analógica de dor, visual, para membros inferiores, induzida pelo exercício; Escala de Borg de esforço percebido; Concentrações de lactato no sangue, pós-exercício. Os resultados obtidos pelo grupo que recebeu a acupuntura: altas pontuações na Escala de Borg (perceptiva de esforço), o que significa que a acupuntura promoveu um maior esforço durante o exercício, sem um aumento da probabilidade de desempenho negativo 
além da diminuição do tempo de conclusão da prova.

Em um estudo mais recente, ${ }^{26}$ foi avaliado salto máximo com queda bídepe e força máxima isométrica do quadríceps voluntária; eletromiografia de superfície para medir a atividade eletromiográfica do músculo reto femoral durante 30 segundos sustentado, dos extensores do joelho. Dividiram 33 atletas recreacionais, sendo 13 do gênero feminino e 20 do gênero masculino, em divididos em 3 grupos (os que receberam a acupuntura sistêmica dos pontos E36, BP6, VC6, Ear Point 55; um grupo de acupuntura simulada em pontos não existentes; e um grupo controle, placebo, de laser desativado).

Obtiveram como resultado um aumento não significante da altura do salto máximo com queda bídepe, comparado o tratamento com acupuntura com acupuntura placebo e grupo controle; aumento significante da força máxima isométrica do quadríceps.

Uma pesquisa $^{27}$ com 12 homens saudáveis estudantes de educação física, com idade entre 19 e 25 anos, avaliou os efeitos da acupuntura auricular nos níveis de ácido lático sanguíneo, induzido pelo exercício $\left(\mathrm{VO}^{2} \mathrm{max}\right)$ em esteira ergométrica. Utilizaram os pontos Fígado, Pulmão, Subcórtex, Glândulas Endócrinas, Triplo Aquecedor da acupuntura auricular. Esses estudantes tiveram como resultado um aumento da captação de oxigênio e diminuição dos níveis de ácido láctico sanguíneo pós-exercício.

As pesquisas ${ }^{9,25}$ apresentaram que o atleta que usa do tratamento de acupuntura, pode atingir melhoras na performance física, com melhoras no tempo de término da prova, sendo isso muito importante para um atleta de alto rendimento. Nos dois trabalhos foram usados pontos do meridiano do Pulmão, sendo P1, ponto Mo do pulmão e P11, ponto Ting, esse órgão tem como função controlar o Qi e a respiração, comunicar e regular as vias dos líquidos e controlar a difusão e descida, essa função significa que o pulmão controla o Qi de todo o corpo e dirige a respiração.

A vesícula biliar aparece com os pontos VB30 E VB34, respectivamente nos dois estudos, a vesícula na MTC (Medicina Tradicional Chinesa) está ligada ao fígado onde 
se armazena a bile com finalidade de ajudar o estomago e o baço/pâncreas na digestão, e também se relaciona com fatores emocionais como transtornos mentais, logo se o atleta respira bem, com boa circulação dos líquidos, boa digestão e mente tranqüila com certeza terá bons resultados.

Os trabalhos realizados com acupuntura auricular ${ }^{23-24,27}$ apresentaram excelentes resultados. Mostraram ganhos no aumento da captação de oxigênio e diminuição de acidose sanguínea, usando os pontos Shen Men, coração, fígado, pulmão, triplo aquecedor, subcórtex e hipófise.

\section{CONSIDERAÇÕES FINAIS}

A acupuntura mostrou ser efetiva para os atletas, tanto no tratamento com acupuntura sistêmica, como auricular, as duas técnicas mostraram resultados semelhantes, melhorando as atividades metabólicas e fisiológicas da população estudada.

São necessários mais estudos que aprofundem os resultados da acupuntura em atletas e suas diferentes modalidades e com outras terapias dentro da Medicina Tradicional Chinesa, como eletroacupuntura, moxabustão e ventosas, pois está claro que a acupuntura pode melhorar o desempenho dos atletas.

\section{REFERÊNCIAS}

${ }^{1}$ WEN, T. S. Acupuntura clássica chinesa. São Paulo: Cultrix, 1985.

${ }^{2}$ CESANA, J. Educação Física e práticas corporais alternativas: o trabalho com o corpo em questão. Motriz, Rio Claro, v. 14, n. 4, p. 462-470, 2005.

${ }^{3} \mathrm{HOPWOOD}$, V. et al. Acupuntura e técnicas relacionadas a fisioterapia. São Paulo: Manole, 2001.

${ }^{4}$ ZHANG, R. Accidents in acupuncture treatment: history and current state. Zhong Xi Yi Conexões: revista da Faculdade de Educação Física da UNICAMP, Campinas, v. 11, n. 3, p. 176-191, jul./set. 2013. ISSN: 1983-9030 
Jie Xue Bao, v. 2, n. 4, p. 306-313, 2004.

${ }^{5}$ PEREIRA, F. A. O. Evidências científicas da ação da acupuntura. Perspectivas, v. 4, n.7, p. 88-105, 2005.

${ }^{6}$ CARNEIRO, N. M. Fundamentos da acupuntura médica. Curitiba: Sistema, 2001.

${ }^{7}$ AKIMOTO, T. et al. Acupuncture and responses of imunologic and endocrine markens during competition. Medicine and Science in Sports and Exercise, Hagerstown, v. 35, n. 8, p. 1296-1302, 2003.

${ }^{8}$ LUZ, M. T. Estudo comparativo das medicinas ocidental contemporânea, homeopática, tradicional chinesa e ayurvédica em programas públicos de saúde. Rio de Janeiro: Universidade do Estado do Rio de Janeiro, Instituto de Medicina Social, 1996.

${ }^{9}$ SANTOS, V. G. et al. Acupuntura na melhora da performance em atletas juvenis de handebol. Saúde e Pesquisa, Maringá, v. 1, n. 3, p. 331-335, 2008.

${ }^{10}$ LUNA, M. P. et al. Efeitos da acupuntura na performance de atletas velocistas de alto rendimento do Rio de Janeiro. Fitness \& Performance, Rio de Janeiro, v. 4, n. 4, p. 199-214, 2005.

${ }^{11}$ SILVA, A. F. G. et. al. Tratamento fisioterapêutico por meio da acupuntura nas lombalgias. RUBS, v. 1, n. 2, p. 1-50, 2005.

${ }^{12}$ SUN, K. O. et al. Acupuncture for frozen shoulder. Hong Kong Medical Journal, v. 7, 2001.

${ }^{13}$ BRUM, K. N.; ALONSO, A. C.; BRECH, G. C. Tratamento de massagem e acupuntura em corredores recreacionais com síndrome do piriforme. Arquivo Ciência e Saúde, v. 16, n. 2, p. 62-66, 2009.

Conexões: revista da Faculdade de Educação Física da UNICAMP, Campinas, v. 11, n. 3, p. 176-191, jul./set. 2013. ISSN: 1983-9030 
${ }^{14}$ MEYER, H. Acupuntura e letargia nos esportes. Bahia: Presscolor Gráficos Especializados, 1992.

${ }^{15}$ SCOGNAMILLO-SZABÓ, M.V. et. al. Acupuntura: bases científicas e aplicações. Ciência Rural, Santa Maria, v. 31, n. 6, p.1091-1099, 2001.

${ }^{16}$ PETERSON, R. P. Mecanismo e etiologia das lesões. In: PETERSON, L.; RENSTROM, P. Lesões do esporte: prevenção e tratamento. São Paulo: Manole, 2002.

${ }^{17}$ PELHAM, T.W. et al. Acupuncture in human performance. Journal of Strength and Conditioning Research, Colorado Springs, v. 5, n. 2, p. 266-271, 2001.

${ }^{18}$ FRANCA, D. et. al. Acupuntura cinética como efeito potencializador dos elementos moduladores do movimento no tratamento de lesões desportivas. Fisioterapia Brasil, Rio de Janeiro, v. 5, n. 2, p. 111-118, 2004.

${ }^{19}$ LEE, S.H. et al. Effects of acupuncture on the 5-hydroxytryptamine synthesis and tryptophan hydroxylase expression in the dorsal raphe of exercises rats. Neurosciense Letters, Limerick, v. 332, n. 1, p. 17-20, 2002.

${ }^{20}$ MYAMOTO, T. Acupuncture treatment for muscle injury. Japanese Journal of Physical Fitness and Sports Medicine, v. 43, n. 2, p. 39-41, 1997.

${ }^{21}$ HABER, D.E.P. Et al. Influence of acupuncture on physical performance capacity and haemodynamic parameters. International Journal of Sports Medicine, Stuttgart, v. 13, n. 6, p. 486-491, 1992.

${ }^{22}$ BOOP-LIMOGE, C. L' acupuncture permet-elle d' ameliorer les performances sportives etude personnelle a propos de 35 sportifs de hault niveau. These Medicine, 1998.

Conexões: revista da Faculdade de Educação Física da UNICAMP, Campinas, v. 11, n. 3, p. 176-191, jul./set. 2013. ISSN: 1983-9030 
${ }^{23}$ LIN, Z. P. et al. Effects of acupuncture stimulation on recovery ability for male elite basketball athletes. American Journal of Chinese Medicine, Garden City, v. 37, n. 3, p. 471-481, 2009.

${ }^{24}$ LIN, Z. P. et al. Effect of auricular acupuncture on oxygen consumption of boxing athletes. Chinese Medical Journal, Peking, v. 22, n. 13, p.1587-1590, 2009.

${ }^{25}$ DHILLON, S. et al. The acute effect of acupuncture on $20-\mathrm{km}$ cycling performance. Clinical Journal of Sport Medicine, New York, v. 18, n. 1, p. 76-80, 2008.

${ }^{26}$ HUBSCHER, M. et al. Immediate effects of acupuncture on strength performance: a ramdomized, controlled crossover trial. European Journal of Applied Physiology, Heidelberg, v. 110, n. 2, p. 353-358, 2010.

${ }^{27} \mathrm{GENG}, \mathrm{L}$. J. et al. Investigation on the effects of ear acupressure on exercise-induced lactic acid levels and the implications for athletic training. American Journal of Acupuncture, Felton, v. 23, n. 4, 1995.

Recebido em: 22 mar. 2013 Aceito em: 02 ago. 2013

Contato: Daniel Vicentini de Oliveira d.vicentini@hotmail.com 